\title{
DPSCs and SHED in Tissue Engineering and Regenerative Medicine
}

\author{
Muhammad Fawwaz Abdullah ${ }^{1,2}$, Kannan Thirumulu Ponnuraj ${ }^{1,3}$ and Khairani Idah Mokhtar ${ }^{1, *}$ \\ ${ }^{1}$ School of Dental Sciences, Health Campus, Universiti Sains Malaysia, 16150 Kubang Kerian, Kelantan, Malaysia \\ ${ }^{2}$ Advanced Medical \& Dental Institute (AMDI), Universiti Sains Malaysia, 13200, Penang, Malaysia \\ ${ }^{3}$ Human Genome Centre, School of Medical Sciences, Health Campus, Universiti Sains Malaysia, 16150 Kubang \\ Kerian, Kelantan, Malaysia
}

\begin{abstract}
Dental pulp stem cells (DPSCs) from permanent teeth and stem cells from human exfoliated deciduous teeth (SHED) have attracted tremendous interest recently by playing a major role in tissue engineering and regenerative medicine. However, since stem cell technology is still in its infancy, interdisciplinary cooperation between medicine, basic biological research, nanotechnology and materials science is needed to achieve successful clinical applications. Similar to mesenchymal stem cells, DPSCs and SHED can undergo self-renewal and have multipotent differentiation ability, but unlike other sources of stem cells such as embryonic stem cells, which involves the destruction of human embryo, DPSCs and SHED have limited ethical concerns as they are readily and easily accessible, non-invasive and disposed off naturally. Not only DPSCs and SHED can be used for cell based therapies and bio-artificial tissue constructs but also made to differentiate into other cell types. Here, we discuss on definitions, opportunities, advantages and limitations of DPSCs and SHED in tissue engineering and regenerative medicine.
\end{abstract}

Keywords: Dental stem cells, DPSCs, regenerative medicine, SHED, tissue engineering.

\section{INTRODUCTION}

Stem cells are unspecialized cells that continually reproduce themselves and can differentiate into specialized cells of one or more types [1]. Stem cells can be divided into two major classes: embryonic/fetal stem cells and adult stem cells [1]. Dental-derived stem cells are one of adult stem cells used as the cell sources for tissue engineering and regenerative medicine [2]. Dental pulp stem cells (DPSCs) from permanent teeth are able to regenerate a dentin-pulplike complex that is composed of mineralized matrix with tubules lined with odontoblasts and fibrous tissue containing blood vessels in an arrangement similar to the dentin-pulp complex found in normal human teeth [3]. Stem cells from human exfoliated deciduous teeth (SHED) are highly proliferative, clonogenic cells capable of differentiating into a variety of cell types including neural cells, adipocytes and odontoblasts [4]. Stem cells from other sources such as embryos, cord blood and bone marrow have been harvested for use in tissue engineering. Tissue engineering is defined as an interdisciplinary field that applies the principles of engineering and life sciences toward the development of biological substitutes that restore, maintain or improve tissue or organ function [5]. According to European Commission [6], tissue engineering is the regeneration of biological tissue through the use of cells, with the aid of supporting structures and/or

*Address correspondence to this author at School of Dental Sciences, Health Campus, Universiti Sains Malaysia 16150 Kubang Kerian, Kelantan, Malaysia; Tel: +609-7675837; Fax: +609-7642026;

E-mail: khairani@kk.usm.my biomolecules. Regenerative medicine is defined as an emerging interdisciplinary field of research and clinical applications focused on the repair, replacement or regeneration of cells, tissues or organs to restore impaired function resulting from any cause, including congenital defects, disease, trauma and aging [7]. Tissue engineering is basically, one part of the major components of regenerative medicine which follows the principles of cell transplantation, materials science and engineering towards the development of biological substitutes which can restore and maintain normal function [8]. It is envisaged in future that adult stem cells may be more easily directed towards specific lineages that might be able to give rise to a wider range of tissues following transplantation than embryonic stem cells. The advantages of DPSCs and SHED enable it to be efficiently exploited to be implemented in tissue engineering and regenerative medicine.

\section{STEM CELLS}

Stem cells were first studied by Becker et al. in 1963 [9] who injected bone marrow cells into irradiated mice and noticed that nodules developed in the spleens of the mice in proportion to the number of bone marrow cells injected. They concluded that each nodule arose from a single marrow cell and found that bone marrow cells were capable of infinite self-renewal, a central characteristic of stem cells. Hence, scientists concluded that stem cells by definition have two essential properties, i.e. the capacity of selfrenewal, giving rise to more stem cells and the capacity to differentiate into different cell lineages under appropriate conditions. Following this, report on the growth of inner cell 


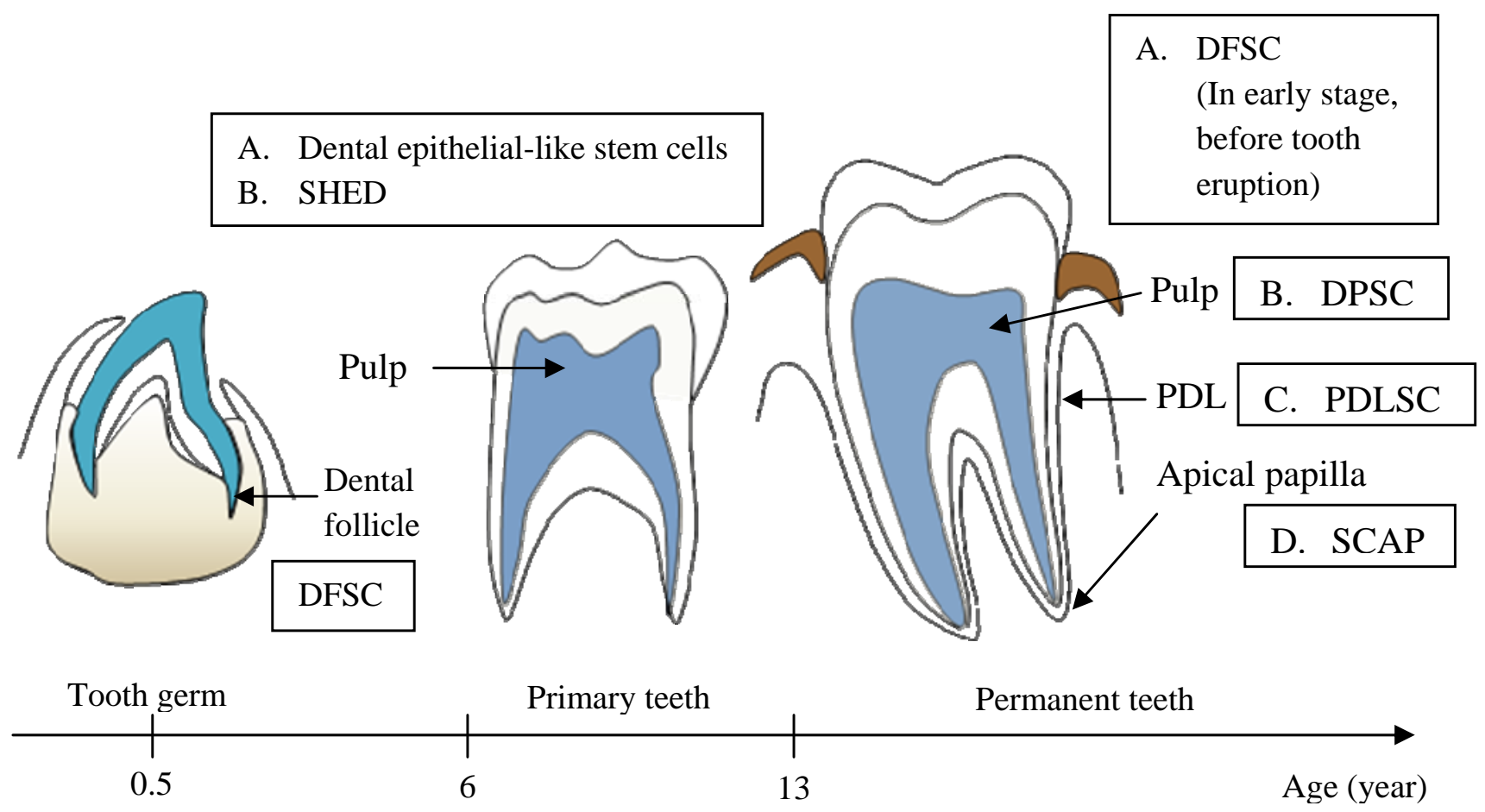

Tooth development processes

Fig. (1). Tooth developmental stages and the derivation of dental-derived stem cells. DFSC $=$ dental follicle stem cells; SHED = stem cells from human primary exfoliated deciduous teeth; DPSC $=$ dental pulp stem cells; PDLSC $=$ periodontal ligament stem cells; SCAP $=$ stem cells from apical papilla.

masses and the isolation of stem cells from human blastocysts were published by Bongso et al. [10]. Later, embryonic stem cell lines were successfully produced for the human [11]. Broadly speaking, there are two main types of stem cells, embryonic and non-embryonic. Embryonic stem cells (ESCs) are totipotent and accordingly, they can differentiate into all three embryonic germ layers. On the other hand, non-ESCs also known as adult stem cells are just multipotent and their potential to differentiate into different cell types seems to be more limited [12], but adult stem cells have much potency with advanced facilities of isolating and expanding thus making them invaluable properties for regenerative medicine.

\section{DENTAL STEM CELL NICHES}

Teeth develop by a series of epithelial-mesenchymal interactions [13]. Interactions between the ectoderm and underlying mesenchyme constitute a central mechanism regulating the morphogenesis of tooth. Tooth morphogenesis is an advancing process that is regulated by sequential and reciprocal interactions between the epithelial and mesenchymal tissues and, during which the simple oral ectoderm thickens, buds, grows and folds to form the complex shape of the tooth crown [14]. Dental stem cells can be divided into dental mesenchymal stem cells (MSCs) that will form the odontoblasts, cementoblasts, osteoblasts and fibroblasts of the periodontal ligament, and dental epithelial stem cells (EpSCs) that give rise to ameloblasts (Fig. 1) [2]. MSCs from human dental tissues include DPSCs in human permanent teeth [3, 15, 16], SHED from deciduous teeth [4], periodontal ligament stem cells (PDLSCs) [17], dental follicle stem cells (DFSCs) from human third molars [18] and stem cells from the apical papilla (SCAP) [19]. Several studies in animals describe the use of EpSCs which have been isolated from newborn or juvenile animals from the third molar teeth. The epithelia were removed and the cells were dissociated enzymatically. However, to implement the same in children is difficult as it would warrant donation of tooth from children [20]. MSCs are thought to reside in a perivascular niche in the dental pulp [21]. All these cells probably share a common lineage of being derived from neural crest cells and all have generic mesenchymal stem cell-like properties, including expression of marker genes and differentiation into mesenchymal cell lineages (osteoblasts, chondrocytes and adipocytes) in vitro and, to some extent, in vivo. The different cell populations do, however, differ in certain aspects of their growth rate in culture, marker gene expression and cell differentiation, although the extent to which these differences can be attributed to tissue of origin, function or culture conditions remains unclear [22].

\section{DENTAL PULP STEM CELLS}

Stem cells isolated from adult human dental pulp were termed DPSCs [3]. They were isolated from permanent third molars and exhibited high proliferation and high frequency of colony formation that produced sporadic, but densely calcified nodules. DPSCs can be collected from dental pulp by means of a non-invasive practice that can be performed in the adult during life after simple extraction and in the young after surgical extraction of wisdom teeth, a routine surgical 
practice plus with less ethical issues $[3,4,23]$. More recently a subpopulation of dental pulp stem cells has been described as human immature dental pulp stem cells (IDPSC) [24]. DPSCs and other adult stem cells can survive for long periods because these cells are quiescent (non-dividing) and reside in a specific perivascular microenvironment where they maintain their stem cell characteristics for long periods of time until they are activated by a normal need for more cells to maintain tissues, or by disease or tissue injury [25]. On the other hand, mesenchymal stem cells (MSCs) derived from bone marrow is not always acceptable owing to the high degree of viral exposure and the significant decrease in the cell number and the proliferative/differentiation capacity along with age [26].

Typical surface markers of MSCs are CD44, CD73, CD90, CD105, CD271 and STRO-1, while the negative markers are CD34, CD45, and HLA-DR [27, 28]. But, there is no distinct marker characterizing DPSCs, which are a heterogeneous and mixed population. DPSCs obtained by their high proliferative potential lean to include a large population cells expressing CD44+, CD90+, and CD166+ [27]. Most of these DPSCs express MSC markers including STRO-1 and CD146 and undergo colony forming in vitro and can regenerate the dentin/pulp complex in vivo [15]. Every researcher may establish different surface markers in each DPSCs population; hence not all the surface markers are expressed in DPSCs population. Generally, most DPSCs show pluripotent stem cells characteristic but exact properties might differ from each other [27].

DPSCs differentiate into functionally active neurons and implanted DPSCs induce endogenous axon guidance, suggesting their potential as cellular therapy for neuronal disorders [29]. Interestingly, in contrast to bone marrow mesenchymal stem cells (BMMSCs), DPSCs do not form areas of active hematopoiesis and adipocyte accumulation at the transplantation site [30]. In another study, when DPSCs were seeded onto human dentin surface and implanted into immunocompromised mice, reparative dentin-like structure was deposited on the dentin surface [16]. These results together raise the possibility that a protocol for pulp tissue regeneration and new dentin formation for clinical therapeutic purposes could be established. Later, Laino and co-workers isolated a selected sub-population of DPSCs called stromal bone producing (SBP) DPSCs and found that these SBP/DPSCs display a great capability of selfexpanding and differentiating in osteoblast precursors (CD44+/RUNX-2+) producing, in vitro, a living autologous fibrous bone (LAB) tissue, which is markedly positive for several bone antibodies [31]. DPSCs were also found to undergo chondrogenic and myogenic differentiation in vitro $[28,31]$. The plasticity and multipotential capability of DPSCs can be explained by the fact that dental pulp is made of both ectodermic and mesenchymal components, containing neural crest-derived cells [28]. After a dental injury, dental pulp is involved in a process called reparative dentinogenesis, where cells elaborate and deposit a new dentin matrix for the repair of the injured site [32]. It has been shown that adult dental pulp contains precursors capable of forming odontoblasts under appropriate signals $[4,15,33$ 34]. Tooth repair is a process which occurs throughout life which suggests that MSCs might exist in adult dental pulp [22]. The in vivo therapeutic targeting of these adult stem cells remains to be explored.

\section{STEM CELLS FROM HUMAN EXFOLIATED DE- CIDUOUS TEETH}

SHED have the capacity to induce bone formation, generate dentine and differentiate into other non-dental mesenchymal cell derivatives in vitro [4, 35-38]. SHED exhibit higher proliferation rates compared to DPSCs and bone marrow-derived MSCs [39], increased population doublings, osteoinductive capacity in vivo, [40, 41], can retain their stem cell characteristics after prolonged culture [42], and an ability to form neural-like spheres in a medium optimized for neural stem cells in vitro [38]. SHED seeded onto tooth slices/scaffolds and implanted subcutaneously into immunodeficient mice differentiated into functional odontoblasts capable of generating tubular dentine and angiogenic endothelial cells [37]. When SHED was transplanted into immunocompromised mice, dentin-like structure was formed which was immunoreactive to dentin specific sialophosphoprotein antibody. Odontoblast-like cells were found to be associated with this regenerated dentin structure, which indicate the odontogenic differentiation potential of SHED [43]. However, unlike DPSCs, SHED did not form a dentin-pulp complex after in vivo transplantation [4]. This indicates that SHED has different odontogenic differentiation potential than DPSCs. With regard to the osteogenic differentiation potential, SHED was different from DPSCs. They were unable to differentiate into osteoblast or osteocyte, but able to induce the host cells to undergo osteogenic differentiation. This observation demonstrates that SHED has an osteoinductive potential rather than a differentiation potential $[4,40,41]$. Therefore, SHED appear to be distinct from the DPSCs and indicate a more immature form than DPSCs. Studies using SHED as a tool in dental pulp tissue engineering in vivo have revealed that the tissue formed when SHED seeded in biodegradable scaffolds prepared within human tooth slices are transplanted into immunodeficient mice has architecture and cellularity closely resembling that of dental pulp, a tissue important for tooth vitality [36]. SHED and other dental stem cells are derived from cranial neural crest ectomesenchyme, and so developmentally and functionally would appear identical, but studies have shown that they do differ and have different gene expression profiles [27]. SHED could therefore be used as a generic allogeneic source of MSCs [22]. SHED were found to express early mesenchymal stem cell markers (STRO-1 and CD146) [2], embryonic stem cell markers (Oct4, NANOG), stage-specific embryonic antigens (SSEA-3, SSEA-4), and tumor recognition antigens (TRA-1-60 and TRA-1-81) [24, 27]. Like DPSCs, SHED also showed the capacity of osteogenic, adipogenic differentiation [43], expressed different neuronal and glial cell markers such as nestin [4, 43]. Even though SHED is used as autologous cells, yet, it is currently restricted to children who have not yet lost all their deciduous teeth [22]. Commercial banking of SHED is thus becoming widespread to enable them to be used once the child becomes an adult. Hence, isolation of postnatal stem cells from an easily accessible is an essential criterion in tissue engineering process. 


\section{TISSUE ENGINEERING FOR REGENERATIVE MEDICINE}

In the past, mankind has been replacing the missing body parts with inanimate objects that were created by him. But with advancing research in biotechnology, regenerative capability of our own living body tissue has opened up a new era of tissue engineering [44]. In 1933, Biscgelie introduced the concept of tissue engineering when mouse tumour cells survived in a biocompatible polymer membrane [45]. Thenceforth, they were implanted into the abdominal cavity of chick embryos. Earlier, in the 1970s, W.T. Green, a paediatric orthopaedic surgeon at Boston's Children's Hospital, carried out a number of experiments to generate new cartilage by implanting chondrocytes seeded on bone spicules in nude mice. He was unsuccessful, but his experiments were part of the first attempts at what we now know as tissue engineering. He had already concluded that, with the advent of biomaterials science, it would be possible to regenerate and produce new tissues by loading viable cells onto 'smart' engineered scaffolds [46]. Tissue engineering strategies can be characterized into two categories: the use of acellular scaffolds, which depend on the body's natural ability to regenerate for proper orientation and direction of new tissue growth, and the use of scaffolds seeded with cells. Acellular tissue matrices are collagen-rich matrices which remove cellular components from tissues via mechanical and chemical manipulation $[47,48]$. Later, these matrices slowly degrade on implantation and are replaced by the extra cellular matrix (ECM) proteins secreted by the ingrowing cells [48]. Tissue engineering approaches to the regeneration of functional tissue using postnatal stem cells can be visualized by three different scenarios: (1) expansion of a population ex vivo before transplantation into the host; (2) re-creation of a tissue or organ ex vivo for transplantation; and (3) design of substances and/or devices for in vivo activation of stem cells, either local or distant, to induce appropriate tissue repair. In all these cases, considerable knowledge of the stem cell population's dynamics is required to predict and control their activity under a variety of different circumstances [8].

\section{ROLES OF DPSCS AND SHED IN REGENERATIVE MEDICINE}

When the tooth is damaged but still in a reparable condition, regeneration of parts of the tooth structure can prevent or delay the loss of the whole tooth. This fact is of importance because tooth loss affects not only basic mouth functions but aesthetic appearance and quality of life [49]. The regenerative response of teeth to damage and structural degeneration are diverse and compartment-dependent, since teeth, as complex structures, harbour both living (periodontal ligament, cementum, pulp) and acellular (enamel, dentine) tissues. The dental pulp plays a major role in tooth regeneration after injury, by participating in a process called reparative dentinogenesis [50]. When pulp tissue is exposed as consequence of the loss of the overlaying dentin, direct pulp-capping therapy allows the pulp to form new dentin [22]. It has been observed that the use of various cementbased compounds, such as mineral trioxide aggregate (MTA) [51], and calcium hydroxide, promotes the activity of cells that remain in the healthy portion of the pulp migrate to the injured site, proliferate by the growth factors released from surrounding dentin matrix and attach the necrotic layer to form osteodentin [52]. Later, the cells attached to osteodentin differentiate into odontoblasts to produce tubular dentin, thus forming reparative dentine [53, 54]. This early mineralized tissue preserves the pulp integrity and serves as protective barrier upon the injury [52]. Yet, regenerative endodontics which is a biologically based procedure designed to replace damaged structures such as dentin, root structures and cells of the pulp-dentin complex [55] will have to depend on tissue engineering therapies to regenerate pulp dentin tissue. The proposed therapies involving stem cells, growth factors, and tissue engineering all require pulp revascularization, which itself is an enormous challenge. One of the most challenging aspects of developing a regenerative endodontic therapy is to understand how the various component procedures can be optimized and integrated to produce the outcome of a regenerated pulp-dentin complex [55].

When the tooth is almost damaged, dentin regeneration becomes difficult as it requires a healthy pulp. Thus, bigger traumas such as tooth avulsion or advanced caries are clinically treated with root canal therapy, in which the entire pulp is cleaned out and replaced with a gutta-percha filling. However, living pulp is critical for the maintenance of tooth homeostasis and essential for tooth longevity [40]. An ideal form of therapy might consist of regenerative approaches in which diseased or necrotic pulp tissues are removed and replaced with regenerated pulp tissues to revitalize the teeth. In particular, the regenerative pulp therapy would reconstitute the normal tissue continuum at the pulp-dentine border by regulating the tissue-specific processes of reparative dentinogenesis [22]. A regenerated pulp tissue should be functionally competent: it should be vascularised, contain similar cell density and architecture of ECM to those of natural pulp, be capable of giving rise to new odontoblasts lining against the existing dentin surface, produce new dentin and be innervated [21]. SHED and DPSCs have potential in dental pulp regeneration.

The first step to engineer tissues is to isolate cells with the right phenotype and propagate them in suitable culturing environments. Prescott et al. demonstrated that DPSCs were able to regenerate pulp-like tissue after being seeded on collagen scaffold supplemented with dentin matrix protein (DMP-1) [56]. Human IDPSC were demonstrated to show significant engraftment in golden retriever muscular dystrophy (GRMD) dog muscles but human dystrophin expression was modest and limited to several muscle fibers [57]. Other studies on DPSCs demonstrated that these cells responded to neuronal inductive conditions both in vitro and in vivo and acquired a neuronal morphology and expressed neuronalspecific markers at both the gene and protein levels [15, 58]. It has been previously reported that multipotential DPSCs retain their neural crest properties following ex vivo expansion [3, 21, 59].

DPSCs also help cardiac repair after myocardial infarction $[43,60]$. The left coronary artery was ligated in nude rats and DPSCs were transplanted to the border of the infarction zone. Evidence of cardiac repair was noted by improved cardiac function, increase in the number of vessels and a 
reduction in infarct size after four weeks transplantation. The cardiac repair occurred in the absence of any evidence of DPSCs differentiation into cardiac or smooth muscle cells. This suggests that DPSCs induced cardiac repair due to its secretion of different growth factors and cytokines such as vascular endothelial growth factor, insulin-like growth factor-1 and -2 and stem cell factor, which helped in inducing angiogensis and cardiac regeneration at the infarction zone $[43,60]$ seeded SHED onto synthetic biodegradable scaffolds and were able to differentiate into odontoblast-like and endothelial-like cells.

DPSCs and SHED also have been shown to induce new end bulb formation and the growth of hairs when associated with upper follicle epithelium [61]. It has also been demonstrated that both types of stem cells were able to generate nerve and brain tissue [4, 27, 62]. SHED was reported to enhance wound healing in an excisional wound-splinting mouse model [63]. Another interesting clinical application has been suggested by investigations of the therapeutic efficacy of SHED in alleviating Parkinson's disease (PD) [38], Alzheimer's disease and cerebral palsy [4, 35, 57, 64, $65]$.

\section{CONCLUSION}

The ease of harvesting, non-involvement of bioethical issues due to their source of origin [3, 4], and expansion of DPSCs and SHED for cell therapies and bio-artificial tissue constructs has widened the horizon of regenerative medicine and tissue engineering [2, 57, 60-63, 66, 67]. Logically, these kinds of procedures are safe and affordable in the future and the restorative procedures to regenerate enamel, dentin and pulp will become available for clinical application. However, one of the important considerations will certainly be the cost of these procedures especially in introducing such technology to clinicians and students from obtaining the dental stem cells to banking and making it available at affordable prices. Similarly, both DPSCs and SHED can also be made to differentiate into the desired cell type in vitro for future use [68]. Hence, the peak of the outcome of DPSCs and SHED in regenerative medicine will rely on the success of the ability of these stem cells to produce a functional pulp tissue for future uses.

\section{CONFLICT OF INTEREST}

The authors confirm that this article content has no conflicts of interest.

\section{ACKNOWLEDGEMENTS}

This study has been approved by Research and Ethics Committee (Human), USM Health Campus USMKK/PPP/JEPeM(211.3[12]) dated $7^{\text {th }}$ April, 2009 and revised on $22^{\text {nd }}$ June, 2010 . This study was done under the USM Short term grant (Number 304/PPSG/6139051).

\section{REFERENCES}

[1] Bongso A, Richards M. History and perspective of stem cell research. Best Pract Res Clin Obstet Gynaecol 2004; 18: 827-42.

[2] Huang YH, Yang JC, Wang CW, Lee SY. Dental stem cells and tooth banking for regenerative medicine. J Exp Clin Med 2010; 2: $111-7$.
[3] Gronthos S, Mankani M, Brahim J, Robey PG, Shi S. Postnatal human dental pulp stem cells (DPSCs) in vitro and in vivo. Proc Natl Acad Sci USA 2000; 97: 13625-30.

[4] Miura M, Gronthos S, Zhao M, et al. SHED - Stem cells from human exfoliated deciduous teeth. J Dent Res 2003; 82: B305-B.

[5] Langer R, Vacanti JP. Tissue engineering. Science 1993; 260: 920 6.

[6] European Commission. Opinion on The State of the Art Concerning Tissue Engineering, The Scientific Committee on Medicinal Products and Medical Devices. SANCO/SCMPMD/2001/0006 Final; 2001.

[7] Greenwood HL, Singer PA, Downey GP, Martin DK, Thorsteinsdottir H, Daar AS. Regenerative medicine and the developing world. PLoS Med 2006; 3: e381.

[8] Bajada S, Mazakova I, Richardson JB, Ashammakhi N. Updates on stem cells and their applications in regenerative medicine. J Tissue Eng Regen Med 2008; 2: 169-83.

[9] Becker AJ, Mc CE, Till JE. Cytological demonstration of the clonal nature of spleen colonies derived from transplanted mouse marrow cells. Nature 1963; 197: 452-4.

[10] Bongso A, Fong CY, Ng SC, Ratnam S. Isolation and culture of inner cell mass cells from human blastocysts. Hum Reprod 1994; 9 : 2110-7.

[11] Thomson JA, Itskovitz-Eldor J, Shapiro SS, et al. Embryonic stem cell lines derived from human blastocysts. Science 1998; 282: 1145-7.

[12] Lee EH, Hui JHP. The potential of stem cells in orthopaedic surgery. J Bone Joint Surg Br 2006; 88B: 841-51.

[13] Tucker AS. Tooth morphogenesis and patterning: molecular genetics. In: eLS. Chichester: John Wiley \& Sons Ltd 2009.

[14] Thesleff I. Epithelial-mesenchymal signalling regulating tooth morphogenesis. J Cell Sci 2003; 116: 1647-8.

[15] Gronthos S, Brahim J, Li W, et al. Stem cell properties of human dental pulp stem cells. J Dent Res 2002; 81: 531-5.

[16] Batouli S, Miura M, Brahim J, et al. Comparison of stem-cellmediated osteogenesis and dentinogenesis. J Dent Res 2003; 82: 976-81.

[17] Seo BM, Miura M, Sonoyama W, Coppe C, Stanyon R, Shi S. Recovery of stem cells from cryopreserved periodontal ligament. J Dent Res 2005; 84: 907-12.

[18] Handa K, Saito M, Tsunoda A, et al. Progenitor cells from dental follicle are able to form cementum matrix in vivo. Connect Tissue Res 2002; 43: 406-8.

[19] Sonoyama W, Liu Y, Fang D, et al. Mesenchymal stem cellmediated functional tooth regeneration in swine. Plos One 2006; 1 e79.

[20] Cherian E, Nandhini G, Kurian A, Rajkumar K. Stem Cells. 1 ed. India: Jaypee Brothers Medical Pub 2011.

[21] Shi S, Bianco P, Robey PG, Gronthos S. Perivascular niche of postnatal mesenchymal stem cells in human bone marrow and dental pulp. J Bone Miner Res 2002; 17: S446-S.

[22] Volponi AA, Pang Y, Sharpe PT. Stem cell-based biological tooth repair and regeneration. Trends Cell Biol 2010; 20: 715-22.

[23] d'Aquino R, De Rosa A, Laino G, et al. Human dental pulp stem cells: from biology to clinical applications. J Exp Zool B Mol Dev Evol 2009; 312B: 408-15.

[24] Kerkis I, Kerkis A, Dozortsev D, et al. Isolation and characterization of a population of immature dental pulp stem cells expressing OCT-4 and other embryonic stem cell markers. Cells Tiss Org 2006; 184: 105-16.

[25] Arianna M, Giuseppe N, Federica S. In: Atwood C, Ed. Embryonic stem cells - recent advances in pluripotent stem cell-based regenerative medicine. Croatia, InTech 2011; pp. 21-48.

[26] Malgieri A, Kantzari E, Patrizi MP, Gambardella S. Bone marrow and umbilical cord blood human mesenchymal stem cells: state of the art. Int J Clin Exp Med 2010; 3: 248-69.

[27] Kawashima N. Characterisation of dental pulp stem cells: A new horizon for tissue regeneration? Arch Oral Biol 2012; 57: 1439-58.

[28] d'Aquino R, Graziano A, Sampaolesi M, et al. Human postnatal dental pulp cells co-differentiate into osteoblasts and endotheliocytes: a pivotal synergy leading to adult bone tissue formation. Cell Death Differ 2007; 14: 1162-71.

[29] Arthur A, Shi S, Zannettino AC, Fujii N, Gronthos S, Koblar SA. Implanted adult human dental pulp stem cells induce endogenous axon guidance. Stem Cells 2009; 27: 2229-37. 
[30] Zavan B, Bressan E, Sivolella S, et al. In: Pignatello R, Ed. Biomaterials Science and Engineering. Croatia, InTech 2011; pp. 339-48.

[31] Laino G, d'Aquino R, Graziano A, et al. A new population of human adult dental pulp stem cells: a useful source of living autologous fibrous bone tissue (LAB). J Bone Miner Res 2005; 20 : 1394-402.

[32] Mitsiadis TA, Rahiotis C. Parallels between tooth development and repair: conserved molecular mechanisms following carious and dental injury. J Dent Res 2004; 83: 896-902.

[33] About I, Mitsiadis TA. Molecular aspects of tooth pathogenesis and repair: in vivo and in vitro models. Adv Dent Res 2001; 15: 59-62.

[34] Tecles O, Laurent P, Zygouritsas S, et al. Activation of human dental pulp progenitor/stem cells in response to odontoblast injury. Arch Oral Biol 2005; 50: 103-8.

[35] Shi S, Bartold PM, Miura M, Seo BM, Robey PG, Gronthos S. The efficacy of mesenchymal stem cells to regenerate and repair dental structures. Orthod Craniofac Res 2005; 8: 191-9.

[36] Cordeiro MM, Dong Z, Kaneko T, et al. Dental pulp tissue engineering with stem cells from exfoliated deciduous teeth. J Endod 2008; 34: 962-9.

[37] Sakai VT, Zhang Z, Dong Z, et al. SHED differentiate into functional odontoblasts and endothelium. J Dent Res 2010; 89: 791-6.

[38] Wang J, Wang X, Sun Z, Yang H, Shi S, Wang S. Stem cells from human-exfoliated deciduous teeth can differentiate into dopaminergic neuron-like cells. Stem Cells Dev 2010; 19: 1375-83.

[39] Nakamura S, Yamada Y, Katagiri W, Sugito T, Ito K, Ueda M. Stem cell proliferation pathways comparison between human exfoliated deciduous teeth and dental pulp stem cells by gene expression profile from promising dental pulp. J Endod 2009; 35: 1536-42.

[40] Machado E, Fernandes MH, Gomes Pde S. Dental stem cells for craniofacial tissue engineering. Oral Surg Oral Med Oral Pathol Oral Radiol 2012; 113: 728-33.

[41] Rosa V, Della Bona A, Cavalcanti BN, Nor JE. Tissue engineering: from research to dental clinics. Dent Mater 2012; 28: 341-8.

[42] Govindasamy V, Ronald VS, Totey S, et al. Micromanipulation of culture niche permits long-term expansion of dental pulp stem cells--an economic and commercial angle. In Vitro Cell Dev Biol Anim 2010; 46: 764-73.

[43] Jamal M, Chogle S, Goodis H, Karam SM. Dental stem cells and their potential role in regenerative medicine. J Med Sci 2011; 4: 5361.

[44] Bhat A, Prasad K, Balaji SS, Bhat A. Role of tissue engineering in dentistry. JIADS 2011; 2: 37-42.

[45] Kim KM, Evans GRD. Tissue engineering: the future of stem cells. In: Ashammakhi H, Reis RL, Eds. Topics in Tissue Engineering. Stem cells 2005; 1-21.

[46] Green WT Jr. Articular cartilage repair. Behavior of rabbit chondrocytes during tissue culture and subsequent allografting. Clin Orthop Relat Res 1977; 154: 237-50.

[47] Chen F, Yoo JJ, Atala A. Acellular collagen matrix as a possible "off the shelf" biomaterial for urethral repair. Urology 1999; 54 : 407-10.

[48] Olson JL, Atala A, Yoo JJ. Tissue engineering: current strategies and future directions. Chonnam Med J 2011; 47: 1-13.

[49] Selwitz RH, Ismail AI, Pitts NB. Dental caries. Lancet 2007; 369: 51-9.

[50] Smith AJ, Cassidy N, Perry H, Begue-Kirn C, Ruch JV, Lesot H. Reactionary dentinogenesis. Int J Dev Biol 1995; 39: 273-80.

[51] Aeinehchi M, Eslami B, Ghanbariha M, Saffar AS. Mineral trioxide aggregate (MTA) and calcium hydroxide as pulp-capping agents in human teeth: a preliminary report. Int Endod J 2003; 36 225-31.

[52] Nakashima M. Bone morphogenetic proteins in dentin regeneration for potential use in endodontic therapy. Cytokine Growth Factor Rev 2005; 16: 369-76.

[53] Schroder U. Effects of calcium hydroxide-containing pulp-capping agents on pulp cell migration, proliferation, and differentiation. $\mathrm{J}$ Dent Res 1985; 64: 541-8.

[54] Tziafas D. The future role of a molecular approach to pulp-dentinal regeneration. Caries Res 2004; 38: 314-20.

[55] Murray PE, Garcia-Godoy F, Hargreaves KM. Regenerative endodontics: a review of current status and a call for action. J Endod 2007; 33: 377-90.

[56] Prescott RS, Alsanea R, Fayad MI, et al. In vivo generation of dental pulp-like tissue by using dental pulp stem cells, a collagen scaffold, and dentin matrix protein 1 after subcutaneous transplantation in mice. J Endod 2008; 34: 421-6.

[57] Kerkis I, Ambrosio CE, Kerkis A, et al. Early transplantation of human immature dental pulp stem cells from baby teeth to golden retriever muscular dystrophy (GRMD) dogs: Local or systemic? J Transl Med 2008; 6: 35.

[58] Davidson RM. Neural form of voltage-dependent sodium current in human cultured dental pulp cells. Arch Oral Biol 1994; 39: 613-20.

[59] Shi S, Gronthos S, Mankani M, Brahim J, Robey PG. Comparison of human dental pulp cells (DPCs) and human bone marrow stromal cells (BMSCs) by in vitro and in vivo differentiation. $\mathrm{J}$ Bone Miner Res 2000; 15: S501-S.

[60] Gandia C, Arminan A, Garcia-Verdugo JM, et al. Human dental pulp stem cells improve left ventricular function, induce angiogenesis, and reduce infarct size in rats with acute myocardial infarction. Stem Cells 2008; 26: 638-45.

[61] Reynolds AJ, Jahoda CA. Cultured human and rat tooth papilla cells induce hair follicle regeneration and fiber growth. Differentiation 2004; 72: 566-75.

[62] Nosrat IV, Smith CA, Mullally P, Olson L, Nosrat CA. Dental pulp cells provide neurotrophic support for dopaminergic neurons and differentiate into neurons in vitro; implications for tissue engineering and repair in the nervous system. Eur J Neurosci 2004; 19: 2388-98.

[63] Nishino Y, Yamada Y, Ebisawa K, et al. Stem cells from human exfoliated deciduous teeth (SHED) enhance wound healing and the possibility of novel cell therapy. Cytotherapy 2011; 13: 598-605.

[64] Arthur A, Rychkov G, Shi S, Koblar SA, Gronthos S. Adult human dental pulp stem cells differentiate toward functionally active neurons under appropriate environmental cues. Stem Cells 2008; 26: 1787-95.

[65] Perry BC, Zhou D, Wu X, et al. Collection, cryopreservation, and characterization of human dental pulp-derived mesenchymal stem cells for banking and clinical use. Tissue Eng Part C Methods 2008; 14: 149-56.

[66] Hara K, Yamada Y, Nakamura S, Umemura E, Ito K, Ueda M. Potential characteristics of stem cells from human exfoliated deciduous teeth compared with bone marrow-derived mesenchymal stem cells for mineralized tissue-forming cell biology. J Endod 2011; 37: 1647-52.

[67] Graziano A, d'Aquino R, Laino G, Papaccio G. Dental pulp stem cells: a promising tool for bone regeneration. Stem Cell Rev 2008; 4: 21-6.

[68] Huang GT, Gronthos S, Shi S. Mesenchymal stem cells derived from dental tissues vs. those from other sources: their biology and role in regenerative medicine. J Dent Res 2009; 88: 792-806.

(C) Abdullah et al.; Licensee Bentham Open.

This is an open access article licensed under the terms of the Creative Commons Attribution Non-Commercial License (http://creativecommons.org/licenses/by-nc/3.0/) which permits unrestricted, non-commercial use, distribution and reproduction in any medium, provided the work is properly cited. 